\title{
Evaluación de Características en Planta, Tubérculo y Rendimiento para Progenies de Semilla Sexual de Papa (Solanum tuberosum), en Valles Altos del Centro de México
}

\author{
Jorge L Sorquis ${ }^{1}$, Francisco J. López ${ }^{1}$
}

\begin{abstract}
Resumen
La producción de papa en México enfrenta problemas de escasez, costo elevado y baja calidad de los tubérculos semilla. Una alternativa es el empleo de semilla sexual de papa (SSP). Este trabajo evaluó el comportamiento de SSP para la obtención de material de siembra uniforme y con buen rendimiento. Resultados de tres ciclos de evaluación proyectan el uso de SSP como viable, al alcanzar altas calificaciones para uniformidad de tubérculo en forma, tamaño y color. Se obtuvieron $1.8 \mathrm{~kg} / \mathrm{m}^{2}$ en la progenie TS9xTPS113 y entre 1.6 y $1.7 \mathrm{~kg} / \mathrm{m}^{2}$ en las progenies MFIIxTS10, LT8xTS9, MFIIxTS9, LT9xTS9 y ChiquitaxTS9. Cuando se sembraron los tubérculos producidos por plantas de SSP los rendimientos fueron en general mucho mayores, incrementándose el de LT9xTS9 a $3.7 \mathrm{~kg} / \mathrm{m}^{2}$, a $3.6 \mathrm{~kg} / \mathrm{m}^{2}$ el de LT8xTS9, a $3.3 \mathrm{~kg} / \mathrm{m}^{2}$ el de Serrana x TS5, y a $3.1 \mathrm{~kg} / \mathrm{m}^{2}$ el de HPS 25/67. La segunda generación de tubérculos semillas mostró rendimiento similar al de la primera generación. El análisis estadístico reveló diferencias significativas para parámetros relacionados con porte de planta y calidad de tubérculo, resultando las progenies antes mencionadas como las más promisorias para usarse en producción de tubérculos semillas.
\end{abstract}

Palabras claves adicionales: $\quad$ papa, semilla sexual de papa, progenie, rendimiento, evaluación.

Aceptado para publicación: setiembre 1998.

${ }^{1}$ Departamento de Biología, ENEP-Iztacala, UNAM. Tlalnepantla, 54090, Estado de México, México.

Email: sarquis@servidor.unam.mex. Fax: 525-565-1009.

Los autores agradecen el invaluable apoyo financiero y logístico a este trabajo por parte las siguientes entidades: Centro Internacional de la Papa, Instituto de Capacitación Agrícola, Forestal y Pecuaria del Estado de México, Centro de Biotecnología SABRITAS y ViVi-Toluca, productora de semilla tubérculo. 


\title{
Evaluation of Plant, Tuber and Yield Characteristics for True Potato (Solanum tuberosum) Seed Progenies in the High Valleys of Central México
}

\begin{abstract}
Summary
Potato production in México faces scarcity, high cost and low quality of seed tubers. An alternative may be at hand in the use of true potato seed (TPS). This research evaluated the performance of TPS for the production of seed tubers of acceptable uniformity and high yield. Results from three cycles of evaluation suggest that the use of TPS is adequate; some progenies scored high for uniformity of tuber color, shape and size. Some of the progenies showed an acceptable yield, TS9xTPS113 yielded $1.8 \mathrm{~kg} / \mathrm{m}^{2}$ while for MFIIxTS10, LT8xTS9, MFIIxTS9, LT9xTS9 and ChiquitaxTS9 yield ranged between 1.6 and $1.7 \mathrm{~kg} / \mathrm{m}^{2}$. When we planted tubers produced from TPS, yields were generally much higher, increasing up to $3.6 \mathrm{~kg} / \mathrm{m}^{2}$ in LT9XTS9, to $3.7 \mathrm{~kg} / \mathrm{m}^{2}$ in LT8XTS9, to $3.3 \mathrm{~kg} / \mathrm{m}^{2}$ in Serrana $\times$ TS5, and to $3.1 \mathrm{~kg} / \mathrm{m}^{2}$ in HPS 25/67. Yields of second generation seedling tubers were not different from those of first generation. The statistical analysis revealed significant differences for several of the characteristics which will determine the final quality and acceptance of TPS for the production of high quality, low cost seed tubers.
\end{abstract}

Additional keywords:

Potato, true potato seed (TPS), progeny, yield, evaluation

\section{Introducción}

Los esquemas convencionales de producción de semilla certificada de papa requieren de elaborados y costosos programas que dependen de la importación periódica de materiales libres de virus provenientes de Holanda, Canadá o los Estados Unidos. Esto sitúa a esta semilla fuera del alcance de miles de pequeños agricultores, quienes tradicionalmente cultivan papa en lotes pequeños de 0.1 a 10 
ha bajo condiciones de temporal. En efecto, aún los grandes productores de papa en los estados del Norte de México como Chihuahua, Sonora, Baja California y Tamaulipas, sufren de un endémico desabastecimiento de semilla.

En total, se cultivan de 70,000 a 100,000 ha de papa en México anualmente (SAGAR, 1995). Sin embargo, típicamente la industria semillera nacional no cubre más del $20 \%$ de la demanda. Bajo estas circunstancias, el desarrollo de tecnologías alternas para la producción de semilla tubérculo de papa se ha convertido en una prioridad nacional.

Al inicio de la década de los ochenta, el Centro Internacional de la Papa (CIP) inició un esfuerzo a gran escala para promover el uso de la semilla sexual de papa o SSP, programa que ha tenido éxito en países del Sureste Asiático, donde la ausencia de una larga tradición en la producción y consumo de papa, permitió a los investigadores concentrarse básicamente en la cuestión del rendimiento (Malagamba y Cabello, 1996; CIP, 1996). Más tarde, el programa incorporó la selección de parentales y esquemas de cruzamiento dirigidos a la obtención de progenies híbridas uniformes y adaptables a un amplio espectro de nichos agroecológicos (Accatino y Malagamba, 1982; Rueda, 1983; Bryan, 1986). Aunque inicialmente la naturaleza autotetraploide de la planta de papa dificultó grandemente la labor de obtención de parentales uniformes con buenas características de floración, al mismo tiempo que una buena aptitud combinatoria general y características agronómicas aceptables (Peloquin, 1979; Grewal y Singh, 1985; Golmirzaie et al., 1987), actualmente existen híbridos de SSP de cruza simple y variedades de polinización abierta que rinden cuando menos 20 t/ha y poseen características aceptables de uniformidad con respecto a tipo y porte de planta, así como color, tamaño y forma de tubérculo (Singh, et al., 1990; Malagamba, 1995; Palláis, 1995). La dormancia de la SSP es todavía un problema, pero ya se cuenta con un paquete tecnológico para monitorearla y asegurar una germinación y un establecimiento uniforme (Sharma y Chandra, 1982; Singh et al., 1990, Pallais, 1995).

Este trabajo se encaminó a la validación de la tecnología de SSP en México mediante evaluación de una colección de progenies de SSP en diversas localidades del centro de México. Aquí reportamos los resultados de ensayos en los que se evaluó la SSP y dos generaciones de semilla tubérculo obtenida de SSP. 


\section{Materiales y Métodos}

El material biológico incluyó dos clones comerciales, Atlantic y Frito Lay 795, así como una colección de 24 progenies de SSP proporcionadas por el CIP. El trabajo experimental se llevó a cabo en los ciclos de temporal de 1995, 1996 y 1997, en los municipios de San Antonio la Isla, Ocuilan y Villa Victoria, Estado de México, respectivamente. En cada caso, la parcela experimental consistió de 3 surcos de $5 \mathrm{~m}$ a $0.92 \mathrm{~m}$ uno de otro, con las plantas espaciadas a $0.25 \mathrm{~m}$; de modo que hubo 60 plantas por parcela y la parcela fue de $13.8 \mathrm{~m}^{2}$. En cada ciclo, se usó un diseño de parcelas completamente al azar con 3 repeticiones. La siembra de SSP se realizó en bandejas de germinación $(30 \times 18 \times 12 \mathrm{~cm})$ a una densidad de 100 semillas por bandeja en sustrato a base de agrolita y musgo (1:1). Las bandejas se mantuvieron 30 días en invernadero bajo un régimen térmico de $22 / 10^{\circ} \mathrm{C}(\mathrm{D} / \mathrm{N})$ y una intensidad luminosa de $400 \mu \mathrm{E} \cdot \mathrm{m}^{-2} \cdot \mathrm{s}^{-1}$. Durante este tiempo las bandejas se mantuvieron humedecidas mediante riego diario con solución ( $4.5 \mathrm{~g} / \mathrm{l})$ de fertilizante (Peter's) de fórmula 945-11. El transplante se realizó a raíz desnuda sobre el terreno previamente preparado mediante barbecho, rastra y surcado según la práctica convencional en la zona; al momento del transplante se realizó la primera aplicación de fertilizante, cuya dosis total, 200-200$230 \mathrm{~kg}$ se fraccionó en tres porciones, para aplicar todo el fósforo, $50 \%$ del nitrógeno y $50 \%$ del potasio al transplante. Un mes después se hizo la segunda aplicación, con $25 \%$ del nitrógeno y $25 \%$ del potasio y, por último, un mes después se aplicó el resto de los fertilizantes. El control de malezas durante el establecimientro del cultivo se logró con la aplicación preemergente de herbicida Sencor (1.0 kg/ha del producto comercial). El control de plagas se efectuó mediante un calendario de aplicaciones regulares, con una o dos por semana, de mezcla de Thiodan con Folimat y/o Talstar, según recomendaciones del fabricante para control de áfidos y mosquita blanca, principalmente. Para tizón tardío se realizaron sólo tres aplicaciones de Flonex o Ridomil, según recomendaciones del fabricante, a partir de los 30 días después del transplante. En el tercer ciclo las condiciones de clima fueron más propicias para una mayor presión de inoculo, por lo cual se hizo una cuarta aplicación sólo en este caso. En los tres ensayos reportados, el ciclo de cultivo fue de 110 días de siembra a cosecha.

En el segundo y tercer ciclo, cuando se sembraron tubérculos semillas cosechados en el ciclo anterior, éstos fueron previamente seleccionados para sembrar material uniforme de 50 a $60 \mathrm{~g}$ cada tubérculo. El material para siembra se mantuvo almacenado a $4^{\circ} \mathrm{C}$ 
en oscuridad y un mes antes de la siembra se puso a brotar bajo luz difusa a temperatura ambiente. Previo a la siembra el material brotado fue tratado con una mezcla de Tecto 60, Bavistin y Captam 50 según recomendaciones del fabricante para prevenir infecciones.

El primer ciclo se realizó en instalaciones del Centro de Biotecnología Sabritas (CBS), en el municipio de San Antonio, La Isla, Estado de México a 2,840 msnm, $19^{\circ} 30^{\prime}$ latitud Norte, precipitación media anual de $840 \mathrm{~mm}$ y temperatura media de $16.2{ }^{\circ} \mathrm{C}$ durante el ciclo del cultivo. El segundo ciclo se llevó a cabo en un predio particular del municipio de Ocuilan, Estado de México a 2,960 msnm, 19 $30^{\prime}$ latitud Norte, con una precipitación media anual de $1,000 \mathrm{~mm}$ y una temperatura media de $12.0{ }^{\circ} \mathrm{C}$ durante el ciclo de cultivo. El tercer ciclo se llevó a cabo en un predio particular en el municipio de Villa Victoria, a 2,100 msnm, $19^{\circ} 30^{\prime}$ latitud Norte, con una precipitación media anual de $1,000 \mathrm{~mm}$ y una temperatura media de $18.8{ }^{\circ} \mathrm{C}$ durante el ciclo de cultivo. En las 3 localidades se buscó la mayor homogeneidad de terreno y la mayor similitud de condiciones de suelo entre localidades; así, los ensayos se montaron en suelos franco-arenosos, profundos, de textura ligera y estructura granular, con $\mathrm{pH}$ ligeramente ácido (5.3 a 6.1).

\section{Diseño experimenta/ y muestreo:}

Para los tres ensayos se usó el diseño de parcelas completamente al azar con tres repeticiones, por tratarse de localidades homogéneas en suelo y clima. En cada caso, el registro de datos de crecimiento aéreo se hizo siempre tomando en cuenta al menos un tercio del total de plantas de cada parcela, es decir 20 plantas, seleccionadas al azar. Los datos tomados al momento de la cosecha se hicieron tomando en cuenta únicamente plantas con competencia perfecta, es decir las 16 plantas centrales del surco central en cada parcela.

\section{Características evaluadas:}

El registro de datos se realizó en dos etapas: a la madurez fisiológica de la planta se registró porcentaje de sobrevivencia, altura de planta, número de tallos por planta, uniformidad de planta en escala de 1 a 9 , donde 9 es el mejor, y condición sanitaria. A la cosecha se registró uniformidad de tamaño, forma y color del tubérculo (escala 1 a 9 , donde 9 es el mejor), número de tubérculos por planta, peso de tubérculos por planta, peso de tubérculos por unidad de superficie y porcentaje comercial de tubérculos. Este porcentaje se definió como la proporción de tubérculos mayores de $40 \mathrm{~mm}$ o con un peso mayor a80g. 


\section{Resultados y Discusión}

El principal objetivo de este trabajo fue caracterizar el rendimiento de una colección de 24 progenies de semilla sexual de papa provenientes del Centro Internacional de la Papa, mediante el transplante a campo, así como la siembra de tubérculos de primera y segunda generación, para evaluar el potencial de la SSP como alternativa para la producción de tubérculos-semillas de alta calidad sanitaria y de bajo costo en México. Durante tres ciclos agrícolas consecutivos se evaluaron características de planta y de tubérculo a diferentes altitudes.

No se encontró efecto significativo por progenie para ninguna de las siguientes variables: porcentaje de sobrevivencia, altura de planta, número de tallos por planta, uniformidad de planta, condición sanitaria, forma o color de tubérculo (datos no mostrados). El porcentaje de sobrevivencia para todas las progenies fue siempre superior a $90 \%$. Testigos y progenies se comportaron de manera muy semejante, obteniendo todos calificaciones promedio entre 6.6 y 8.5 (en escala de 1 a 9) para uniformidad de planta y tubérculo.

En cambio, se observó efecto significativo por progenie y por ciclo sobre las siguientes variables: tamaño de tubérculo, porcentaje de tubérculo comercial, número de tubérculos por planta, peso de tubérculo por planta y peso de tubérculo $/ \mathrm{m}^{2}$ (Tabla 1).

Tabla 1. Cocientes $F$ de cuadrados medios a cuadrados medios del error obtenidos mediante el análisis de varianza para progenie y ciclo en ensayos uniformes de semilla sexual de papa.

\begin{tabular}{|c|c|c|c|c|c|c|}
\hline \multicolumn{7}{|c|}{ Cocientes F } \\
\hline $\begin{array}{l}\text { Fuente de } \\
\text { variación }\end{array}$ & g.l. & $\begin{array}{l}\text { Tamaño } \\
\text { tubérculo }\end{array}$ & $\begin{array}{l}\text { Tubérculo } \\
\text { comercial }\end{array}$ & $\begin{array}{l}\text { Número } \\
\text { tubérculo/ }\end{array}$ & $\mathrm{kg} / \mathrm{pl}$ & $\mathrm{kg} / \mathrm{m}^{2}$ \\
\hline Progenie & 23 & $111.0 \mathrm{a}$ & $38.53 a$ & $12.96 a$ & $11.37 a$ & $17.27 \mathrm{~b}$ \\
\hline Ciclo & 2 & $64.8 \mathrm{~b}$ & $87.14 b$ & $47.42 \mathrm{NS}$ & $23.49 b$ & 71.51b \\
\hline Prog. x Ciclo & 1 & 25. $8 b$ & $8.61 b$ & $29.18 N S$ & $45.49 b$ & $1.70 b$ \\
\hline
\end{tabular}

La Tabla 2 resume la comparación de medias para los parámetros sobre los cuales se detectó efecto significativo por progenie en el 
primer ciclo, en la localidad de San Antonio la Isla, Estado de México. Como resultado del primer ciclo de ensayo se identificaron siete progenies por sus características favorables en comparación con dos testigos ampliamente usados para la industria de la fritura y en el mercado fresco. Estos testigos son variedades clonales de excelente rendimiento y características agronómicas. Las mejores progenies incluyeron Chiquita x TS9, LT8 x TS9, MFII x TS9, LT9 x TS9, MFII $x$ TS10, TS9 $x$ TPS113 y Achirana $x$ TS4. Como puede verse, cuatro de ellas incluyen a TS9 como progenitor masculino y su rendimiento fue muy semejante. En contraste, otras dos cruzas que incluyen al mismo progenitor masculino, I-1035 x TS9 y MFI x TS9, rindieron muy pobremente y ocuparon los últimos lugares de clasificación. Por otra parte, dos cruzas incluyen a TS9 como progenitor femenino; TS9 $\mathrm{x}$ TS5, la cual tuvo un rendimiento bajo, y TS9 x TPS113, que fue la de mejor rendimiento en la primera generación de transplante a campo. Estos resultados indican que no puede afirmarse nada sobre un clon como progenitor masculino o como progenitor femenino al margen de la cruza específica en cuestión.

Las progenies de SSP registraron amplia variación entre ellas para varias de las características de interés. De ellas, no fue posible distinguir de los testigos cuando menos a dos en uniformidad de tubérculo en forma, tamaño y color, ya que estas dos progenies recibieron calificaciones de las más altas. El rendimiento, sin embargo, no fue comparable al de los testigos, que varió entre 3.3 y $3.6 \mathrm{~kg} / \mathrm{m}^{2}$. En contraste, las mejores progenies de SSP no superaron los $1.8 \mathrm{~kg} / \mathrm{m}^{2}$. En este primer ciclo el porcentaje de tubérculos de tamaño comercial varió entre 18 y $81 \%$, para la progenie con menor proporción (LT9 x TS9) y el testigo con mayor proporción de tubérculo comercial (FL795), respectivamente. Solamente tres de las progenies tuvieron apenas un poco más del $50 \%$ de tubérculos de tamaño comercial (Tabla 2). Cabe destacar que ninguna de las progenies de mayor rendimiento produjo más del $50 \%$ de tubérculos de tamaño comercial. Puesto que el tamaño del tubérculo para semilla no es un criterio limitante, parece ser que las progenies más rendidoras pueden usarse mejor para la producción de tubérculos semillas. Los datos sobre número de tubérculos por planta dan apoyo a esta idea.

La mayoría de las progenies produjo un promedio elevado de tubérculos por planta, la mayoría de ellos de pequeños a medianos y todas produjeron más tubérculos por planta que cualquiera de los dos testigos. Algunas, como TS9 x TPS113 o LT8 x TS9, produjeron más de 14 tubérculos por planta (Tabla 2), es decir, más de $100 \%$ que los testigos. Sin embargo, típicamente sólo 5 ó 6 de estos tubérculos alcanzaron un tamaño comercial, mientras que el resto eran minitubérculos de entre 30 y $60 \mathrm{~g}$ cada uno. Aún aquellas progenies 
de bajo rendimiento produjeron mayor número de tubérculos por planta que los testigos. El número promedio de tubérculos por planta varió entre 8.0 y 15.3 entre progenies en el primer ciclo y no se modificó significativamente en ciclos subsecuentes. Una variación aún mayor se reportó para 14 progenies de SSP comparadas con los cultivares Kennebec y R. Burbank en Washington (Martin, 1983). En ese caso al menos nueve de las progenies evaluadas superaron a los testigos en número de tubérculos por planta.

Durante el segundo ciclo se evaluó la primera generación de tubérculos semillas cosechados de SSP (Tabla 3). Este segundo ciclo permitió reconocer dos cosas. En primer lugar, disminuyó notablemente la variación poblacional para cualquiera de los parámetros evaluados. En este caso, todas las calificaciones otorgadas fueron superiores a las de primer ciclo (datos no mostrados). Esto indica que el producto generado con la siembra de los tubérculos semilla obtenidos de la SSP es más uniforme y homogéneo que el producto proveniente directamente de SSP. En segundo lugar, todos los materiales mostraron rendimientos significativamente superiores a los de primer ciclo y mucho más comparables a los de los testigos comerciales incluidos. Esto coincide con reportes previos de ensayos internacionales de SSP (Gaur and Pandey, 1993) y muestra que el mejor uso que puede darse a la SSP es usarla para la producción de tubérculos semillas. Por ejemplo, en primer ciclo la progenie LT9 x TS9 rindió $1.7 \mathrm{~kg} / \mathrm{m}^{2}$, comparable al rendimiento obtenido en la India, $1.8 \mathrm{~kg} / \mathrm{m}^{2}$ (CIP, 1994). De esto, sólo el $18 \%$ fue de tamaño comercial. En el segundo ciclo, la semilla tubérculo proveniente de la SSP de la misma progenie mostró un rendimiento de casi $3.73 \mathrm{~kg} / \mathrm{m}^{2}$, más del doble que en el primer ciclo y $78 \%$ de esta cosecha fue de tamaño comercial. En el segundo ciclo cuando menos cuatro progenies fueron estadísticamente iguales al mejor de los dos testigos y 12 de las progenies fueron estadísticamente iguales al segundo mejor testigo. El incremento en rendimiento se atribuyó al aumento en peso de los tubérculos por planta más que a una modificación del número de tubérculos por planta (Tabla 3). Ensayos semejantes realizados en otras latitudes han demostrado consistentemente un incremento sustancial del rendimiento y de la uniformidad de planta y tubérculo en la población obtenida con tubérculos semillas de SSP que con esta última, independientemente de fechas de siembra y manejo agronómico del cultivo (Upadhya y Chandra, 1985). Esto ha sido atribuido al mayor vigor que caracteriza a las plantas provenientes de tubérculos semillas en comparación al de las plantas que provienen de SSP. A su vez, esta diferencia en vigor entre ambos tipos de semilla ha sido atribuida a la pérdida de viabilidad del embrión en muchos de los 
materiales en la subespecie tuberosum, precisamente por haberse preferenciado por tanto tiempo la propagación asexual (Upadhya et al., 1982). La selección de materiales con embrión tipo A parece ser eficaz para la obtención de progenies de mayor vigor (Gaur y Pandey, 1993).

Tabla 2. Comparación de medias para componentes del rendimiento de progenies de SSP cultivadas por transplante (Primer ciclo).

\begin{tabular}{|c|c|c|c|c|}
\hline \multirow[b]{2}{*}{ Progenie o cultivar } & \multirow{2}{*}{$\begin{array}{l}\text { (\%) tubérculo } \\
\text { comercial }\end{array}$} & \multirow{2}{*}{$\begin{array}{c}\text { Número } \\
\text { tubérculos/pl. }\end{array}$} & \multicolumn{2}{|c|}{ Peso de tubérculos } \\
\hline & & & kg/pl. & $\mathrm{kg} / \mathrm{m}^{2}$ \\
\hline Frito Lay 795 & $81.7^{a *}$ & $7.4 \mathrm{~cd}$ & $0.795 a$ & $3.3 a$ \\
\hline Atlantic & $65.0 \mathrm{ab}$ & $7.1 \mathrm{~cd}$ & $0.821 a$ & $3.6 a$ \\
\hline HPS 25/67 & $56.2 \mathrm{abc}$ & $9.2 \mathrm{bc}$ & $0.300 \mathrm{de}$ & 0.99 cde \\
\hline Mainex104.12LB & $52.8 \mathrm{bcd}$ & $11.6 a b$ & $0.286 \mathrm{de}$ & 1.0cde \\
\hline Chiquita $\times$ TS9 & $52.0 \mathrm{bcd}$ & 10.3ab & $0.502 b$ & $1.62 b$ \\
\hline HPS $1 / 13$ & $51.9 \mathrm{bcd}$ & $10.5 a b$ & $0.315 \mathrm{de}$ & 1.17cde \\
\hline LT9×104.12LB & $48.6 \mathrm{cde}$ & $9.6 \mathrm{bc}$ & $0.146 f$ & 0.64 efg \\
\hline 1-1 035xTS9 & 48.4cde & $9.8 b c$ & $0.273 d e$ & $1.07 \mathrm{cde}$ \\
\hline Serrana x TS5 & 47.4cde & $10.2 \mathrm{bc}$ & $0.317 \mathrm{de}$ & $1.35 \mathrm{~cd}$ \\
\hline TS9 $\times$ TS5 & $42.0 \mathrm{cde}$ & $10.7 \mathrm{ab}$ & $0.322 \mathrm{de}$ & $1.28 \mathrm{~cd}$ \\
\hline Chiquita $\times$ TS4 & 38.9 cde & $8.6 \mathrm{bcd}$ & $0.203 e f$ & 0.87 cde \\
\hline HPS 7/67 & $38.6 \mathrm{cde}$ & $11.4 a b$ & $0.344 \mathrm{de}$ & $1.44 b c$ \\
\hline LT8 $\times$ TS9 & 36.9def & $15.3 a$ & $0.439 \mathrm{C}$ & $1.69 \mathrm{~b}$ \\
\hline MFIIXTS10 & 36.3def & $12.7 \mathrm{a}$ & $0.422 \mathrm{C}$ & $1.73 b$ \\
\hline LT8x104.12LB & $36.2 \mathrm{def}$ & $8.7 b c$ & $0.158 f$ & $0.75 \mathrm{def}$ \\
\hline HPS $7 / 67$ & 35.6def & $8.5 \mathrm{bcd}$ & 0.236 ef & $1.00 \mathrm{cde}$ \\
\hline Serrana $x$ Kathadin & $34.5 \mathrm{efg}$ & $12.6 a$ & $0.362 \mathrm{cde}$ & 1.41 be \\
\hline MFI x TS9 & 33.9efg & $8.4 \mathrm{bcd}$ & $0.148 f$ & 0.75 def \\
\hline HPS 7/1 3 & 33.3eíg & $9.7 \mathrm{bc}$ & $0.170 f$ & 0.68efg \\
\hline LT8 x LT7 & 29.9 efg & $9.0 b c$ & 0.227 ef & $0.96 \mathrm{cde}$ \\
\hline HPS II/67 & $28.3 f g h$ & 10.7ab & $0.383 \mathrm{~cd}$ & $1.49 b c$ \\
\hline TS9xTPS113 & 27.8fgh & $14.7 \mathrm{a}$ & $0.429 c$ & $1.81 b$ \\
\hline Achirana x TS4 & 25.0fgh & $11.5 a b$ & $0.390 \mathrm{~cd}$ & $1.54 b c$ \\
\hline TS6 $\times$ TPS67 & 23.8ghi & S.Obcd & $0.145 f$ & $0.59 f g$ \\
\hline MFIIxTS9 & 23.3ghi & $13.4 a$ & $0.400 \mathrm{~cd}$ & $1.68 \mathrm{~b}$ \\
\hline LT9 $x$ TS9 & 18.2hi & $11.8 a b$ & $0.423 \mathrm{C}$ & $1.67 \mathrm{~b}$ \\
\hline
\end{tabular}

*Medias seguidas de la misma letra en cada columna no fueron estadísticamente diferentes a 0.05 de probabilidad de acuerdo a la prueba de Turkey. 
Tabla 3. Comparación de medias para componentes del rendimiento de progenies de SSP cultivadas mediante la primera generación de tubérculos (segundo ciclo).

\begin{tabular}{|c|c|c|c|c|}
\hline \multirow[t]{2}{*}{ Progenie o cultivar } & \multirow{2}{*}{$\begin{array}{l}(\%) \\
\text { tubérculo } \\
\text { comercial }\end{array}$} & \multirow{2}{*}{$\begin{array}{l}\text { Número } \\
\text { tubérculos/pl. }\end{array}$} & \multicolumn{2}{|c|}{ Peso de tubérculos } \\
\hline & & & $\overline{\mathrm{kg} / \mathrm{pl} .}$ & $\mathrm{kg} / \mathrm{m}^{2}$ \\
\hline Frito lay 795 & $82 a^{*}$ & $6.8 \mathrm{~cd}$ & $0.865 a b$ & $3.45 a b$ \\
\hline Atlantic & $71 b$ & $7.5 \mathrm{~cd}$ & $0.783 \mathrm{bc}$ & $3.16 \mathrm{bc}$ \\
\hline HPS 25/67 & $65 b c$ & $9.0 \mathrm{bc}$ & $0.754 b c$ & $3.11 \mathrm{bc}$ \\
\hline Mainex104.12LB & $65 b c$ & $12.1 \mathrm{ab}$ & 0.442def & 1.83def \\
\hline Chiquita $\times$ TS9 & $60 \mathrm{bc}$ & 10.0ab & 0.317 ef & 1.31ef \\
\hline HPS $1 / 13$ & 65 be & 10.0ab & 0.450def & 1.80def \\
\hline LT9×104.12LB & $65 b c$ & $8.7 \mathrm{bcd}$ & 0.539def & 2.12def \\
\hline 1-1035 xTS9 & $70 b$ & $9.5 b c$ & $0.392 \mathrm{ef}$ & $1.55 e f$ \\
\hline Serrana x TS5 & $66 b c$ & $9.8 \mathrm{bc}$ & $0.834 a b$ & 3.31ab \\
\hline TS9 $\times$ TS5 & $65 b c$ & 11.0ab & 0.320ef & 1.25í \\
\hline Chiquita x TS4 & $65 \mathrm{bc}$ & $8.5 b c d$ & 0.400 ef & $1.66 \mathrm{ef}$ \\
\hline HPS 7/67 & $75 a b$ & $11.2 \mathrm{ab}$ & $0.624 \mathrm{~cd}$ & $2.52 \mathrm{~cd}$ \\
\hline LT8 $\times$ TS9 & $60 c$ & $11.6 a b$ & $0.923 a$ & $3.60 a$ \\
\hline MFII xTS10 & $78 a b$ & $11.8 a b$ & $0.890 a$ & $3.55 a$ \\
\hline LT8x 104.12LB & $65 b c$ & $7.8 \mathrm{~cd}$ & 0.600 cde & 2.46cde \\
\hline HPS I/67 & $70 b$ & 8.0bcd & 0.518def & 2.00def \\
\hline Serrana x Kathadin & $65 b c$ & $12.3 a$ & $0.280 f$ & $1.12 f$ \\
\hline MFI x TS9 & $63 b c$ & 8.8bcd & $0.656 \mathrm{~cd}$ & $2.66 \mathrm{~cd}$ \\
\hline HPS 7/1 3 & $59 c$ & S.Obcd & $0.673 \mathrm{~cd}$ & $2.75 \mathrm{~cd}$ \\
\hline LT8 x LT7 & $72 \mathrm{bc}$ & $9.1 \mathrm{be}$ & $0.605 \mathrm{cde}$ & 2.47cde \\
\hline HPS U/67 & $68 b c$ & 10.1ab & $0.650 \mathrm{~cd}$ & $2.61 \mathrm{~cd}$ \\
\hline TS9xTPS113 & $60 c$ & $12.3 a$ & 0.467def & 1.95def \\
\hline Achirana x TS4 & $65 b c$ & 11.3ab & $0.650 \mathrm{~cd}$ & $2.64 \mathrm{~cd}$ \\
\hline TS5 x TPS67 & $75 b c$ & $6.7 \mathrm{~cd}$ & $0.335 \mathrm{ef}$ & 1.30ef \\
\hline MFII xTS9 & $65 b c$ & 12.0ab & 0.480def & $1.95 \mathrm{def}$ \\
\hline LT9 $\times$ TS9 & $78 a b$ & $11.2 \mathrm{ab}$ & $0.950 a$ & $3.73 a$ \\
\hline
\end{tabular}

*Medias seguidas de la misma letra en cada columna no fueron estadísticamente diferentes a 0.05 de probabilidad de acuerdo a la prueba de Turkey.

Otra de las progenies que mostró alto rendimiento y buenas características de tubérculo fue LT8 x TS9, que en la primera evaluación registró $1.7 \mathrm{~kg} / \mathrm{m}^{2}$ y $37 \%$ de tamaño comercial, mientras que alcanzó $3.6 \mathrm{~kg} / \mathrm{m}^{2}$ y $60 \%$ de tamaño comercial en el segundo ciclo (Tabla 3). Comparando estos rendimientos con ensayos de tubérculos semillas proveniente de SSP en otros países, resultan muy comparables a los obtenidos en Bangladesh, donde LT8 x TS9 rindió $3.66 \mathrm{~kg} / \mathrm{m}^{2}$. Cabe aclarar que Bangladesh fue el país donde se lograron los mejores rendimientos promedio entre 1986 y 1990 en ensayos internacionales (CIP, 1990). 
Aunque en este trabajo no todas las progenies presentaron un buen rendimiento, debe reconocerse que mucho se ha logrado en la fijación de características de calidad y uniformidad del tubérculo producido por SSP. Se ha encontrado que una de las razones por las que una progenie puede no tener un buen rendimiento es que entre las progenies derivadas de cruzamientos dentro y entre grupos taxonómicos de papa, el grupo más heterocigótico es el de mayor rendimiento y el más estable (Mendoza y Haynes, 1974; Sharma et al., 1988). Así, cabe la posibilidad de que exista en las 24 progenies de SSP evaluadas en este trabajo un porcentaje importante de cruzas que no sean las más aptas.

El tercer ciclo de evaluación mostró que la mayoría de las progenies mantuvieron el nivel de rendimiento observado en el segundo ciclo, lo cual sustenta el argumento anterior respecto a la estabilidad de rendimiento de progenies aptas (Tabla 4). En este caso, cuando menos cuatro progenies fueron estadísticamente iguales a los testigos, que en este caso no difirieron entre sí como en el ciclo anterior. Todas las progenies mostraron cuando menos $60 \%$ de tubérculo comercial y varias superaron el $70 \%$.

La diferencia en rendimientos entre los ciclos segundo y tercero parece haber obedecido más a las diferencias ambientales entre ambos ciclos que a cualquier otra causa. Aunque no se tomaron registros de precipitación y temperatura, pudo observarse que durante el tercer ciclo las temperaturas fueron en general ligeramente menores a causa de un temporal más abundante y mejor distribuido, mientras que en el segundo ciclo se experimentó una leve sequía durante el primer mes después de la siembra. Esto, aunque no causó pérdida de plantas, retrasó levemente el desarrollo del cultivo y por ende, dado que la duración del ciclo fue la misma, afectó al rendimiento.

En conclusión, nuestros resultados corroboran y extienden observaciones previas que permitirán ampliar la base de datos del CIP para comportamiento de progenies de SSP, a efecto de mantener o eliminar una progenie dada de acuerdo con los resultados obtenidos en ensayos uniformes internacionales. Las progenies identificadas aquí para alto rendimiento pueden usarse para producir tubérculos semillas de excelente calidad para la producción comercial de papa en el área de estudio. 
Tabla 4. Comparación de medias para componentes del rendimiento de progenies de SSP cultivadas mediante la segunda generación de semilla tubérculo (Tercer ciclo).

\begin{tabular}{|c|c|c|c|c|}
\hline \multirow[t]{2}{*}{ Progenie o cultivar } & \multirow{2}{*}{$\begin{array}{c}\%) \\
\text { tuhérculo }\end{array}$} & \multirow{2}{*}{$\begin{array}{c}\text { Número } \\
\text { tubércullos/nl. }\end{array}$} & \multicolumn{2}{|c|}{ Peso de tubérculos } \\
\hline & & & kg/pl. & $\mathrm{kg} / \mathrm{m}^{2}$ \\
\hline Frito Lay 795 & $72 a b^{*}$ & $8.4 \mathrm{~cd}$ & $1.130 a b$ & $4.15 a$ \\
\hline Atlantic & $77 b$ & $7.8 \mathrm{~cd}$ & 0.993bc & $3.76 a$ \\
\hline HPS 25/67 & $68 b c$ & 9.1 be & $0.832 b c$ & $3.45 b$ \\
\hline Maine $x$ 104.12LB & $66 b c$ & 10.9ab & 0.354def & $1.44 \mathrm{ef}$ \\
\hline Chiquita x TS9 & $69 b c$ & $11.2 \mathrm{ab}$ & $0.397 \mathrm{ef}$ & $1.57 \mathrm{e}$ \\
\hline HPS $1 / 13$ & $63 b c$ & 10.9ab & 0.568def & $2.37 \mathrm{~cd}$ \\
\hline LT9x 104.12LB & $66 b c$ & $9.8 b c$ & 0.598def & $2.39 \mathrm{~cd}$ \\
\hline $1-1035$ xTS9 & $74 b$ & $10.3 \mathrm{bc}$ & 0.476 ef & $1.85 \mathrm{de}$ \\
\hline Serrana $\times$ TS5 & $70 b c$ & $10.5 b c$ & $0.910 a b$ & $3.50 a b$ \\
\hline TS9xTS5 & $67 \mathrm{bc}$ & $11.8 a b$ & $0.260 f$ & $1.20 f$ \\
\hline Chiquita x TS4 & $64 b c$ & S.Obcd & 0.375 ef & $1.65 \mathrm{e}$ \\
\hline HPS 7/67 & $71 a b$ & $12.2 \mathrm{ab}$ & $0.652 \mathrm{~cd}$ & $2.48 \mathrm{~cd}$ \\
\hline LT8 x TS9 & $67 c$ & $15.4 a$ & $1.016^{a}$ & $3.89 a$ \\
\hline MFHxTS10 & $75 a b$ & $12.0 \mathrm{a}$ & $1.040 a$ & $3.75 a$ \\
\hline LT8×104.12LB & $60 \mathrm{bc}$ & $8.9 b c$ & $0.631 \mathrm{cde}$ & $2.55 \mathrm{C}$ \\
\hline HPS 7/67 & $75 b$ & 8.7bcd & $0.543 d e f$ & $2.35 \mathrm{~cd}$ \\
\hline Serrana $\times$ Kathadin & $60 \mathrm{bc}$ & $12.6 a$ & $0.236 f$ & $1.45 \mathrm{ef}$ \\
\hline MFI x TS9 & $60 \mathrm{bc}$ & 9.0bcd & $0.737 \mathrm{~cd}$ & $3.00 b c$ \\
\hline HPS 7/13 & $64 c$ & $9.5 b c$ & $0.620 \mathrm{~cd}$ & $2.34 \mathrm{~cd}$ \\
\hline LT8 x LT7 & $77 \mathrm{bc}$ & $10.3 b c$ & 0.660 cde & $2.87 b c$ \\
\hline HPS II/67 & $78 b c$ & 10.0ab & $0.550 \mathrm{~cd}$ & $2.31 \mathrm{~cd}$ \\
\hline TS9xTPS113 & $65 c$ & $16.7 \mathrm{a}$ & 0.403def & $1.56 \mathrm{e}$ \\
\hline Achirana $\times$ TS4 & $65 b c$ & $11.5 a b$ & $0.550 \mathrm{~cd}$ & $2.39 \mathrm{~cd}$ \\
\hline TS6 $\times$ TPS67 & $70 b c$ & $9.2 \mathrm{bcd}$ & $0.246 \mathrm{ef}$ & $1.00 f$ \\
\hline MFII xTS9 & $68 b c$ & $15.4 \mathrm{a}$ & 0.412 def & $1.70 \mathrm{e}$ \\
\hline LT9 $\times$ TS9 & $68 a b$ & 12.8ab & $1.150 \mathrm{a}$ & $4.13 a$ \\
\hline
\end{tabular}

*Medias seguidas de la misma letra en cada columna no fueron estadísticamente diferentes a 0.05 de probabilidad de acuerdo a la prueba de Tukey.

\section{Referencias Bibliográficas}

1. Accatino, P., Malagamba, P. 1982. Potato production from true potato seed. International Potato Center Lima, Perú.

2. Bryan, J. 1986. TPS production and distribution by CIP-Lima True Potato Seed Letter, CIP, Vol No.2, 20 p. 
3. Centro Internacional de la Papa. 1986. CIP. Annual Report. CIP, urna. p. 23-33.

4. Centro Internacional de la Papa. 1990. CIP. Annual Report. CIP, Lima, p. 15-41.

5. Centro Internacional de la Papa. 1994. CIP. Annual Report. CIP, Lima, p. 12-36.

6. Gaur, P. C.; Pandey, S.K. 1993. True potato seed (TPS) technology. Pp. 85-111. En: Advances in Horticulture Vol. 7-Potato. K. L. Chadha, J. S. Grewl (eds.). Malhotra Publishing House, India.

7. Grewal, J. S.; Singh, A.N. 1985. Problems and prospects of TPS technology transfer in India. En: Proceedings of Regional Workshop on True potato Seed for Potato Production. NPDP Nepal and CIP (Region V) Kathmandu, Nepal, p. 43-45.

8. Golmirzaie, A.; Bretschneider, M.K.; Ortíz, O. 1987. Inbreeding effect on the production and agronomical character of different true potato seed generations. En: EAPR Abstracts, Aalborg. Denmark, July 2631, 1987. p. 294-295.

9. Malagamba, P. 1995. Producción de papa con semilla sexual. En: Manual de producción de papa: Sistemas de uso de semilla sexual de papa para diferentes ambientes. Unidad Técnica de Capacitación, Fasículo 3.1. p. 5.

10. Malagamba, P.; Cabello, R. 1995. Producción de semilla sexual de papa. Centro Internacional de la Papa Lima. Perú. Fasículo 2.1. p. 36.

11. Martin, M. M. 1983. Field production of potatoes from true seed and its use in a breeding programme. Potato Res. 26: 219-227.

12. Mendoza, H. A. and F. L. Haynes. 1974. Genetic basis of heterosis for yield in autotetraploid potatoes. Theor. Appl. Genet. 45:21-25.

13. Pallais, N. 1995. Producción de papa con semilla sexual. Principios de manejo post cosecha y evaluación de la calidad de la semilla sexual de papa. Centro Internacional de la Papa (CIP). Fascículo 2.4. p. 23.

14. Peloquin, S. J. 1979. Breeding methods for archieving phenotypic uniformity. En: Report of Planning Conference on the production of the potato from true seed. CIP Lima. p. 151 -155.

15. Rueda, J. L. 1983. Breeding for adoption of TPS propagaíion. En: Report of Planning Conference: Utilization of Genetic Resources III. p. 23-40.

16. Secretaría de Agricultura y Ganadería. 1996. Producción de papa por zonas agrícolas de temporal y de riego en 1995. En: Anuario estadístico de la producción agrícola en México. SAGAR. p. 115-121. 
17. Singh, J. P.; Pandey, P.C.; Singh, S.V. . 1990. Production, certification and utilization of TPS parental lines and seedling tubers. En: Commercial Adoption of True Potato Seed Technology-Prospects and Problems (P.C. Gaur, De) Central Potato Research Institute, Shimla. p. 116-119.

18. Sharma, K. P.; Chandra, R. 1982. Prospects of commercial potato with true seed. Indian Farmer's Digest. 15: 13-15.

19. Sharma, K. P.; Katoch, C.; Sharma, S.K.; Rana, M.S. 1988. Genetic analysis for combining ability in $\mathrm{F} 1 \mathrm{C} 1$ clonal generation in potato (Solanum tuberosum L). J. Indian Potato Assoc. 15: 186-189.

20. Upadhya, M. D.; Sharma, N.K.; Garg, K.C. 1982. Studies on the use of the true seed for raising, a commercial potato crop. En: Potato in Developing Countries. B. Nogalch et al. (eds.). Indian Potato Association, Shimla. p. 56-63.

21. Upadhya, M. D.; Chandra, R. 1985. Technology for production of true potato seed. Indian Farming 34:18-21. 